Article

\title{
Tumor Marker-Based Definition of the Transarterial Chemoembolization-Refractoriness in Intermediate-Stage Hepatocellular Carcinoma: A Multi-Cohort Study
}

\author{
Jun Sik Yoon ${ }^{1,2,+}$, Dong Hyun Sinn ${ }^{3,+}$, Jeong-Hoon Lee ${ }^{1, t, * \mathbb{D}}$, Hwi Young Kim $4, * \mathbb{D}$, \\ Cheol-Hyung Lee ${ }^{1}$, Sun Woong Kim ${ }^{1}$ D, Hyo Young Lee ${ }^{1,5}$, Joon Yeul Nam 1,4, Young Chang 1,6, \\ Yun Bin Lee ${ }^{1} \mathbb{D}^{\text {, Eun Ju Cho }}{ }^{1}{ }^{\mathbb{D}}$, Su Jong Yu ${ }^{1}$, Hyo-Cheol Kim ${ }^{7}$, Jin Wook Chung ${ }^{7}$, \\ Yoon Jun $\operatorname{Kim}^{1}$ (D) and Jung-Hwan Yoon ${ }^{1}$ \\ 1 Department of Internal Medicine and Liver Research Institute, Seoul National University College of \\ Medicine, Seoul 03080, Korea; yojusi@naver.com (J.S.Y.); hyung6519@gmail.com (C.-H.L.); \\ withtwohands@gmail.com (S.W.K.); catchhyong@gmail.com (H.Y.L.); moreno777@gmail.com (J.Y.N.); \\ chyoung86@gmail.com (Y.C.); yblee@snu.ac.kr (Y.B.L.); creatioex@gmail.com (E.J.C.); \\ ydoctor2@hanmail.net (S.J.Y.); yoonjun@snu.ac.kr (Y.J.K.); yoonjh@snu.ac.kr (J.-H.Y.) \\ 2 Department of Internal Medicine, Busan Paik Hospital, Inje University College of Medicine, \\ Busan 47392, Korea \\ 3 Department of Internal Medicine, Samsung Medical Center, Seoul 06351, Korea; sinndhn@hanmail.net \\ 4 Department of Internal Medicine, College of Medicine, Ewha Womans University, Seoul 07804, Korea \\ 5 Department of Internal Medicine, Eulji General Hospital, Eulji University School of Medicine, \\ Seoul 01830, Korea \\ 6 Department of Internal Medicine, Digestive Disease Center, Institute for Digestive Research, Soonchunhyang \\ University College of Medicine, Seoul 04401, Korea \\ 7 Department of Radiology, Seoul National University College of Medicine, Seoul 03080, Korea; \\ angiointervention@gmail.com (H.-C.K.); chungjw@snu.ac.kr (J.W.C.) \\ * Correspondence: pindra@empal.com (J.-H.L.); hwiyoung@ewha.ac.kr (H.Y.K.); \\ Tel.: +82-2-2072-2228 (J.-H.L.); +82-2-2650-5868 (H.Y.K.); Fax: +82-2-743-6701 (J.-H.L.); \\ +82-2-2650-2837 (H.Y.K.) \\ $+\quad$ These three authors are co-first authors.
}

Received: 17 September 2019; Accepted: 1 November 2019; Published: 4 November 2019

\begin{abstract}
Background: For patients with hepatocellular carcinoma (HCC), the definition of refractoriness to transarterial chemoembolization (TACE), which might make them a candidate for systemic therapy, is still controversial. We aimed to derive and validate a tumor marker-based algorithm to define the refractoriness to TACE in patients with intermediate-stage HCC. Methods: This multi-cohort study was comprised of patients who underwent TACE for treatment-naïve intermediate-stage HCC. We derived a prediction model for overall survival (OS) using the preand post-TACE model to predict tumor recurrence after living donor liver transplantation (MoRAL) (i.e., MoRAL score $=11 \times \sqrt{ }$ protein induced by vitamin Kabsence-II $+2 \times \sqrt{ }$ alpha-fetoprotein), which was proven to reflect both tumor burden and biologic aggressiveness of HCC in the explant liver, from a training cohort $(n=193)$. These results were externally validated in both an independent hospital cohort (from two large-volume centers, $\mathrm{n}=140$ ) and a Korean National Cancer Registry sample cohort $(\mathrm{n}=149)$. Results: The changes in MoRAL score $(\triangle \mathrm{MoRAL})$ after initial TACE was an independent predictor of OS (MoRAL-increase vs. MoRAL-non-increase: adjusted hazard ratio $(\mathrm{HR})=2.18,95 \%$ confidence interval $(\mathrm{CI})=1.37-3.46, p=0.001$; median $\mathrm{OS}=18.8$ vs. 37.8 months). In a subgroup of patients with a high baseline MoRAL score $(\geq 89.5,25$ th percentile and higher), the prognostic impact of $\triangle$ MoRAL was more pronounced (MoRAL-increase vs. MoRAL-non-increase: $\mathrm{HR}=3.68$, $95 \% \mathrm{CI}=1.54-8.76, p<0.001 ;$ median OS $=9.9$ vs. 37.4 months). These results were reproduced in the external validation cohorts. Conclusion: The $\triangle$ MoRAL after the first TACE, a simple and objective
\end{abstract}


index, provides refined prognostication for patients with intermediate-stage HCC. Proceeding to a second TACE may not provide additional survival benefits in cases of a MoRAL-increase after the first TACE in patients with a high baseline MoRAL score $(\geq 89.5)$, who might be candidates for systemic therapy.

Keywords: tumor marker; alpha-fetoprotein; protein induced by vitamin K absence-II; hepatocellular carcinoma; tumor biology

\section{Introduction}

Hepatocellular carcinoma (HCC) is an aggressive tumor that is the fourth leading cause of cancer-related mortality worldwide [1]. In patients with intermediate-stage (Barcelona Clinic Liver Cancer [BCLC] stage B) HCC, who have multinodular HCC without macrovascular invasion or extrahepatic metastasis, the recommended treatment modality is transarterial chemoembolization (TACE) [2,3]. TACE is performed in a palliative manner, and the decision-making for retreatment with TACE is critical in managing HCC patients. The decision to repeat TACE or switch to other treatment modalities should be determined on the basis of the clinical response of the HCC patient. In cases of TACE refractoriness, the switch from TACE to sorafenib has been reported to improve survival in intermediate-stage HCC patients [4-6].

There have been several decision-making models developed for TACE retreatment, such as the Assessment for Retreatment with TACE (ART) score [7] and the ABCR (standing for alpha-fetoprotein (AFP), BCLC, Child-Pugh, and Response) score [8], which have been controversial in their efficacy in retrospective validation studies [9-12]. Therefore, such scoring systems for TACE retreatment were not adopted in the most recent practice guidelines $[13,14]$. Moreover, the scoring systems (i.e., the ART score and ABCR score) have shown that radiological tumor response plays a key role in this clinical decision. Although the radiological tumor response has a strong prognostic performance after TACE [15-18], the interpretation of the radiological tumor response is complex and heavily depends on the individual radiologist's subjective measurement. As a result, inter-reader disagreement might occur in the radiological tumor response $[19,20]$. Therefore, an objective and reproducible model that can help decision-making for retreatment with TACE is warranted.

Recently, we developed a model to predict tumor recurrence after living donor liver transplantation (LT) (i.e., a model to predict tumor recurrence after living donor liver transplantation (MoRAL score)) using only two HCC-related tumor markers: protein induced by vitamin K absence-II (PIVKA-II) and AFP [21]. The histological analysis of the explant liver showed that the MoRAL score had significant positive correlations (positive Pearson correlation coefficients) with histological prognostic factors (i.e., Edmonson-Steiner nuclear grade, microvascular invasion, perineural invasion, etc.). Moreover, the MoRAL score was also correlated with entire tumor volume (i.e., size and number). The MoRAL score is an objective and reproducible model that can reflect both tumor burden and biological aggressiveness. We hypothesized that an algorithm utilizing the MoRAL score could also provide refined prognostication in patients with intermediate-stage HCC who have a high tumor burden and aggressive tumor biology.

Therefore, in this study, we aimed to derive and validate the MoRAL score-based algorithm for guiding the decision for retreatment with TACE in patients with intermediate-stage HCC.

\section{Results}

\subsection{Clinical Characteristics}

During the study period, among the 745 patients who were admitted to SNUH with treatment-naïve intermediate-stage HCC, 290 patients were eligible for this study and 97 patients were excluded 
according to exclusion criteria (Supplementary Figure S1A). Finally, a total of 193 patients were included as the training cohort. The same inclusion/exclusion criteria were applied in the two other hospitals during the study period and 140 patients were included as the hospital validation cohort (Supplementary Figure S1B). In the Korean Primary Liver Cancer Registry database, among the 10,578 patients who had treatment-naïve HCC, 637 patients were intermediate-stage HCC and were treated with TACE as a first treatment. Among them, 149 patients were included as the nationwide validation cohort (Supplementary Figure S1C).

The characteristics of the three cohorts prior to the first and second TACE, and changes in the prognostic variables, are shown in Table 1. Viral hepatitis was the most common cause of HCC. The majority of patients with chronic hepatitis B in the training cohort (125/132, 94.7\%) and hospital validation cohort $(90 / 99,90.9 \%)$ were treated with nucleos(t)ide analogues, and the rates were not significantly different between the two cohorts ( $p=0.26$ by Chi-square test). The rates of sustained viral response in patients with chronic hepatitis $C$ were also not significantly different between the two cohorts $(25.0 \%$ vs. $33.3 \% ; p=0.70$ by Fisher's exact test). In the training cohort $(\mathrm{n}=193)$, the median MoRAL score decreased from a baseline of $259.2(\mathrm{IQR}=89.8-778.2)$ to 93.8 $(\mathrm{IQR}=60.9-231.4)$. Most patients were classified as MoRAL-non-increase and 33 patients $(17.1 \%)$ were classified as MoRAL-increase between the first and second TACE sessions. The median and mean time intervals between the first and second TACE were 55 days (interquartile range $(\mathrm{IQR})=41.5-70.0$ days) and 57.2 days ( $95 \%$ confidence interval $(\mathrm{CI}), 54.4-60.1$ days), respectively. The median total number of TACE sessions was six (IQR $=4.0-8.0)$.

In the hospital validation cohort $(\mathrm{n}=140)$, the baseline MoRAL score $(237.8, \mathrm{IQR}=93.1-419.3)$ was lower than in the training cohort. Between the first and second TACE, 41 patients (29.3\%) were classified as MoRAL-increase. The median and mean time intervals between the first and second TACE were 35 days ( $\mathrm{IQR}=30.0-43.0$ days) and 39.9 days (95\% CI, 37.5-42.3 days), respectively. The median total number of TACE sessions was five $(\mathrm{IQR}=2.3-8.0)$. In the nationwide validation cohort $(\mathrm{n}=149)$, the baseline MoRAL score (311.9, IQR = 133.0-544.6) was the highest among the three cohorts. The data of tumor markers before the second session of TACE were not available from the database. The median and mean time intervals between the first and second TACE were 50 days (IQR $=34.0-62.0$ days) and 50.1 days (95\% CI, 47.2-53.0 days), respectively. 
Table 1. Clinical characteristics in the three study cohorts.

\begin{tabular}{|c|c|c|c|}
\hline Variables & $\begin{array}{l}\text { Training Cohort } \\
\quad(n=193)\end{array}$ & $\begin{array}{l}\text { Hospital validation } \\
\text { Cohort }(\mathrm{n}=140)\end{array}$ & $\begin{array}{l}\text { Nationwide Validation } \\
\text { Cohort }(\mathrm{n}=149)\end{array}$ \\
\hline \multicolumn{4}{|l|}{ Before the first session of TACE } \\
\hline Age & $63.0(55.0-70.0)$ & $60.0(53.0-68.0)$ & $61.0(54.0-68.0)$ \\
\hline Male sex & $164(85.0 \%)$ & $117(83.6 \%)$ & $127(85.2 \%)$ \\
\hline \multicolumn{4}{|l|}{ Etiology } \\
\hline HBV infection & $132(68.4 \%)$ & 99 (70.7\%) & $96(64.4 \%)$ \\
\hline HCV infection & $24(12.4 \%)$ & $12(8.6 \%)$ & $20(13.4 \%)$ \\
\hline Alcohol & $20(10.4 \%)$ & $20(14.3 \%)$ & $24(16.1 \%)$ \\
\hline Others & $17(8.8 \%)$ & $9(6.4 \%)$ & $9(6.0 \%)$ \\
\hline \multicolumn{4}{|l|}{ Child-Pugh class } \\
\hline A & $164(85 \%)$ & $128(91.4 \%)$ & $125(83.9 \%)$ \\
\hline B 7 points & $18(9.3 \%)$ & $9(6.4 \%)$ & $15(10.1 \%)$ \\
\hline$\geq \mathrm{B} 8$ points & $11(5.7 \%)$ & $3(2.1 \%)$ & $9(6.0 \%)$ \\
\hline Tumor number & $4.0(2.0-5.0)$ & $4.0(2.0-5.0)$ & $4.0(2.0-5.0)$ \\
\hline Maximum tumor size & $5.0(3.3-8.4)$ & $4.6(3.5-7.0)$ & $5.0(3.4-7.0)$ \\
\hline AFP, ng/mL & $38.8(8.2-711.0)$ & $29.0(8.8-529.2)$ & $59.6(14.6-874.1)$ \\
\hline PIVKA-II, mAU/mL & $358.0(48.0-3499.0)$ & $288.0(41.5-1200.0)$ & $693.0(88.0-2000.0)$ \\
\hline MoRAL score & $259.2(89.5-780.6)$ & $237.8(93.1-419.3)$ & $311.9(133.0-544.6)$ \\
\hline \multicolumn{4}{|l|}{ Before the second session of TACE } \\
\hline AFP, ng/mL & $15.2(5.3-85.9)$ & $14.4(6.2-120.0)$ & - \\
\hline PIVKA-II, mAU/mL & $42(24-236)$ & $53.8(22.0-450.0)$ & - \\
\hline MoRAL score & $93.8(60.9-231.4)$ & $103.2(62.7-263.8)$ & - \\
\hline Radiological tumor response ${ }^{\mathrm{a}}$ & & & - \\
\hline Absent (SD/PD) & $55(28.5 \%)$ & $44(31.4 \%)$ & \\
\hline Present (PR) & $138(71.5 \%)$ & $96(68.6 \%)$ & \\
\hline \multicolumn{4}{|l|}{ Dynamics of variables } \\
\hline Child-Pugh increase & & & - \\
\hline Absent & $145(75.1 \%)$ & $108(77.1 \%)$ & \\
\hline+1 point & $43(22.3 \%)$ & $30(21.4 \%)$ & \\
\hline+2 points or more & $5(2.6 \%)$ & $2(1.4 \%)$ & \\
\hline AST increase $>25 \%$ & $22(11.4 \%)$ & $26(18.6 \%)$ & - \\
\hline$\triangle \mathrm{MoRAL}$ & & & - \\
\hline MoRAL-increase & $33(17.1 \%)$ & $41(29.3 \%)$ & \\
\hline MoRAL-non-increase & $163(82.9 \%)$ & 99 (70.7\%) & \\
\hline
\end{tabular}

Abbreviations: TACE-transarterial chemoembolization; HBV-hepatitis B virus; HCV-hepatitis C virus; AFP-alpha-fetoprotein; PIVKA-II — protein induced by vitamin K absence-II; MoRAL—model to predict tumor recurrence after living donor liver transplantation; SD—stable disease; PD—progressive disease; PR—partial response; AST-aspartate aminotransferase. ${ }^{a}$ Evaluated before the second session of TACE according to EASL criteria.

\subsection{Derivation of Prognostic Models from the Training Cohort}

In the training cohort, 106 patients (54.9\%) died during the study period (median follow-up duration $=47.4$ months, $\mathrm{IQR}=31.8-62.5$ months) and the median OS was 26.1 months (IQR $=16.0-40.0$ months). Among the baseline and post-TACE variables, the baseline Child-Pugh class, radiological tumor response, worsening in Child-Pugh score between the first and second sessions of TACE, and $\triangle \mathrm{MoRAL}$ had significant associations with OS in a univariable analysis (all log-rank $p<0.05$, Table 2). The risk of death was significantly higher in patients with a MoRAL-increase than in those with a MoRAL-non-increase (hazard ratio $(\mathrm{HR})=1.96,95 \% \mathrm{CI}=1.24-3.09, p=0.003)$. The median OS was 18.8 months $(95 \%$ $\mathrm{CI}=9.9-32.8$ months) in the MoRAL-increase patients and 37.8 months (95\% CI $=31.8-45.6$ months) in the MoRAL-non-increase patients (Figure 1A). However, the baseline MoRAL score and serum levels of the respective tumor markers (i.e., AFP and PIVKA-II) had no significant associations with OS. There was a significant correlation between each radiological tumor response (PR, SD, or PD) and the $\triangle$ MoRAL percentage (Spearman $r=0.23, p=0.001$; Supplementary Figure S2). To avoid statistical redundancy, we performed a multivariable analysis separately using the radiological tumor response (model 1) and $\triangle$ MoRAL (model 2), along with the significant predictive variables from the univariable analysis. In model 1, the adjusted HR (aHR) of the absence of radiological PR was 1.63 $(95 \% \mathrm{CI}=1.08-2.46, p=0.019$; Table 3). In model 2, the aHR of MoRAL-increase was $2.18(95 \% \mathrm{CI}=$ 
$1.37-3.46, p=0.001)$. Although there was no statistically significant difference, patients with alcoholic liver disease tended to have a shorter median OS than patients with other causes ( 25.3 months vs. 39.3 months, $p=0.10$ ). Therefore, in order to avoid possible bias from including patients with alcoholic liver disease, we conducted a Cox regression analysis in a subgroup excluding 20 patients with alcoholic liver disease. In the subgroup analysis, the aHR of MoRAL-increase was 2.06 (95\% CI, 1.27-3.35, $p=0.004)$, which was similar to that of the entire population (Supplementary Table S1).

Table 2. Univariable analysis of prognostic factors in the training cohort $(n=193)$.

\begin{tabular}{|c|c|c|c|c|c|}
\hline \multirow{2}{*}{ Variables } & \multirow{2}{*}{ Group Analysis } & \multicolumn{3}{|c|}{ Overall Survival (Months) } & \multirow{2}{*}{$p$ Value } \\
\hline & & $n=193$ & Median & $95 \% \mathrm{CI}$ & \\
\hline \multirow[t]{2}{*}{ Age } & $<65$ & 109 & 37.8 & $26.9-45.6$ & \\
\hline & $\geq 65$ & 84 & 32.5 & $26.9-42.9$ & 0.685 \\
\hline \multirow[t]{2}{*}{ Sex } & Male & 164 & 33.3 & $26.9-42.1$ & \\
\hline & Female & 29 & 44 & $29.9-61.5$ & 0.331 \\
\hline \multirow[t]{2}{*}{ Etiology } & Viral & 156 & 39.3 & $32.1-45.6$ & \\
\hline & Other & 37 & 26.9 & $19.7-33.5$ & 0.150 \\
\hline \multirow{2}{*}{ Child-Pugh class } & A & 151 & 39.5 & $32.5-45.6$ & \\
\hline & $\mathrm{B}$ & 42 & 23.9 & $17.8-32.8$ & 0.020 \\
\hline \multirow[t]{2}{*}{ Tumor number } & $<4$ & 86 & 40.2 & $28.4-61.5$ & \\
\hline & $\geq 4$ & 107 & 32.8 & $26.6-39.5$ & 0.086 \\
\hline \multirow[t]{2}{*}{ Tumor size, $\mathrm{cm}$} & $<5$ & 95 & 40.2 & $27.5-46.6$ & \\
\hline & $\geq 5$ & 98 & 32.8 & $26.9-39.5$ & 0.847 \\
\hline \multirow{2}{*}{$\mathrm{AFP}, \mathrm{ng} / \mathrm{mL}$} & $<200$ & 125 & 33.3 & $28.4-60.7$ & \\
\hline & $\geq 200$ & 68 & 34.3 & $25.6-42.9$ & 0.229 \\
\hline \multirow[t]{2}{*}{ PIVKA-II, mAU/mL } & $<500$ & 105 & 37.8 & $28.4-46.6$ & \\
\hline & $\geq 500$ & 88 & 33.5 & $25.8-43.0$ & 0.991 \\
\hline \multirow{2}{*}{$\begin{array}{l}\text { Baseline MoRAL } \\
\text { score }\end{array}$} & $<314.8$ & 105 & 37.8 & $28.4-46.6$ & \\
\hline & $\geq 314.8$ & 88 & 32.7 & $25.3-43.0$ & 0.689 \\
\hline \multirow{2}{*}{$\begin{array}{l}\text { Radiological tumor } \\
\text { response }^{\mathrm{b}}\end{array}$} & Absent (SD/PD) & 55 & 26.9 & $18.5-33.2$ & \\
\hline & Present (PR) & 138 & 40.2 & $31.8-46.6$ & 0.014 \\
\hline \multirow[t]{2}{*}{ AST increase $>25 \%$} & Absent & 171 & 37.4 & $31.8-43.0$ & \\
\hline & Present & 22 & 26.9 & $17.8-29.9$ & 0.123 \\
\hline \multirow{2}{*}{ Child-Pugh increase } & Absent & 145 & 42.1 & $33.2-49.1$ & \\
\hline & Present & 48 & 26.9 & $24.0-32.1$ & 0.001 \\
\hline \multirow[t]{2}{*}{$\triangle$ MoRAL } & MoRAL-non-increase & 160 & 37.8 & $31.8-45.6$ & \\
\hline & MoRAL-increase & 33 & 18.8 & $9.9-32.8$ & 0.003 \\
\hline
\end{tabular}

Abbreviations: AFP—alpha-fetoprotein; PIVKA-II—protein induced by vitamin K absence-II; MoRAL-model to predict tumor recurrence after living donor liver transplantation; SD—-stable disease; $\mathrm{PD}$ - progressive disease; PR—partial response; AST—aspartate aminotransferase; TACE—-transarterial chemoembolization; EASL—European Association for the Study of the Liver. ${ }^{a}$ By log-rank test ${ }^{b}$ Evaluated before the second session of TACE according to EASL criteria.

We divided patients in the training cohort using a cutoff of the 25th percentile value of the baseline MoRAL score (i.e., 89.5). In patients with a low baseline MoRAL score $(<89.5), \triangle$ MoRAL was not associated with OS (HR $=0.84,95 \% \mathrm{CI}=0.37-1.92, p=0.69$; Figure 1B). However, in patients with a high baseline MoRAL score ( $\geq 89.5)$, the prognostic impact of $\triangle$ MoRAL was more pronounced than in the whole study population (MoRAL-increase vs. MoRAL-non-increase: $\mathrm{HR}=3.68 ; 95 \% \mathrm{CI}=1.54-8.76$, $p<0.001)$. The median OS times of the MoRAL-increase patients and MoRAL-non-increase patients were 9.9 months (95\% CI $=5.5-24.5$ months) and 37.4 months (95\% CI $=31.0-44.0$ months), respectively (Figure 1C). 

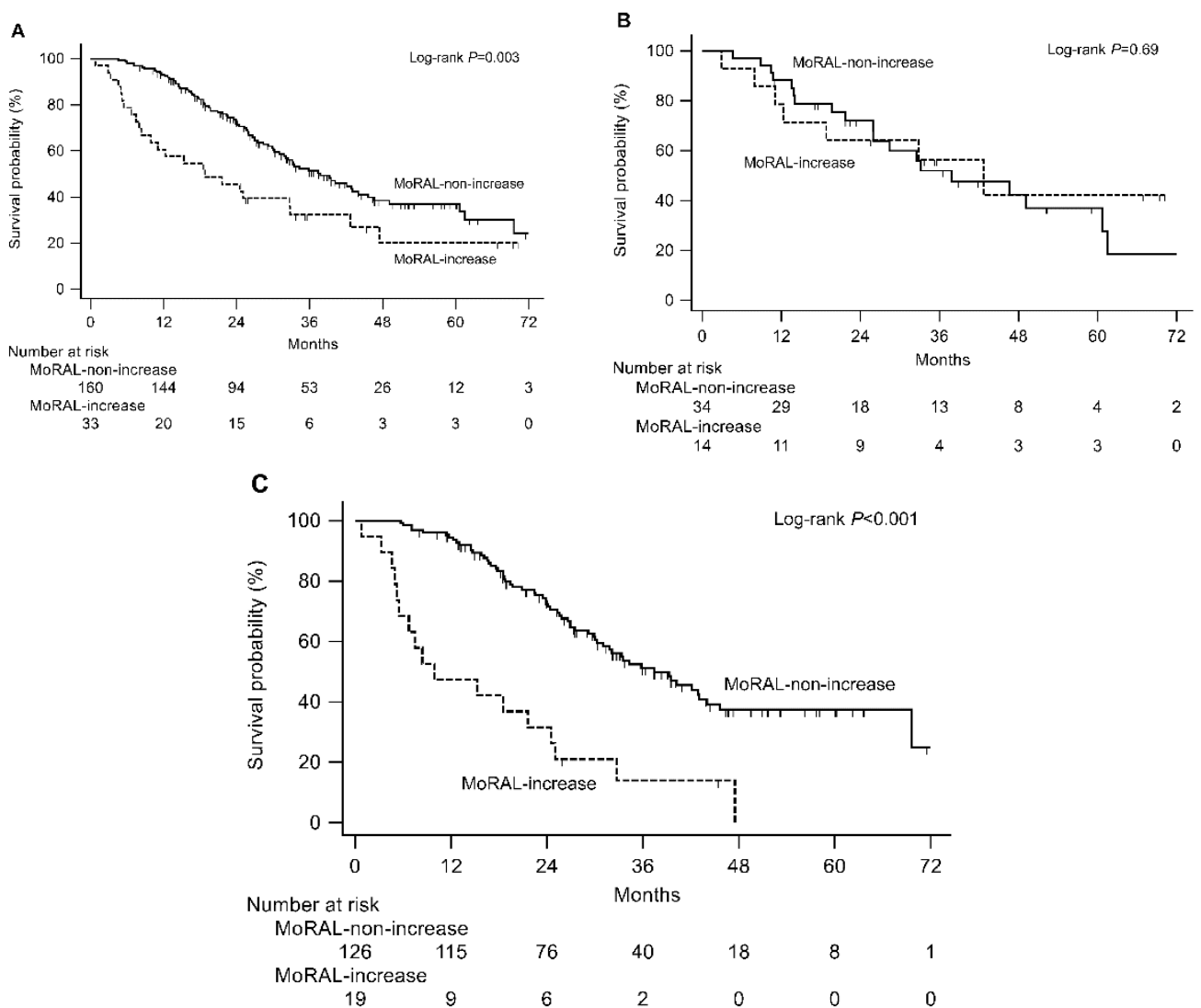

Figure 1. Prognostic significance of $\triangle \mathrm{MoRAL}$ among the entire study population (A) and patients baseline MoRAL score $\leq 89.5$ (B) and $>89.5$ (C) in the training cohort.

Table 3. Multivariable analysis of prognostic factors in the training cohort $(n=193)$.

\begin{tabular}{|c|c|c|c|c|c|c|c|}
\hline \multirow{3}{*}{ Variables } & \multirow{3}{*}{ Group Analysis } & \multicolumn{3}{|c|}{ Model 1} & \multicolumn{3}{|c|}{ Model 2} \\
\hline & & \multicolumn{2}{|c|}{$\begin{array}{l}\text { Overall Survival } \\
\text { (Months) }\end{array}$} & \multirow{2}{*}{$p \underset{\mathbf{a}}{p \text { Value }}$} & \multicolumn{2}{|c|}{$\begin{array}{l}\text { Overall Survival } \\
\text { (Months) }\end{array}$} & \multirow{2}{*}{$p \underset{\mathbf{a}}{p \text { Value }}$} \\
\hline & & $\begin{array}{l}\text { Hazard } \\
\text { Ratio }\end{array}$ & $95 \% \mathrm{CI}$ & & $\begin{array}{l}\text { Hazard } \\
\text { Ratio }\end{array}$ & $95 \%$ CI & \\
\hline \multirow[t]{2}{*}{ Child-Pugh class } & A & 1 (Ref) & - & - & 1 (Ref) & - & - \\
\hline & B & 1.57 & $1.02-2.42$ & 0.039 & 1.59 & $1.04-2.45$ & 0.034 \\
\hline \multirow{2}{*}{$\begin{array}{l}\text { Child-Pugh score } \\
\text { increase }\end{array}$} & Absent & 1 (Ref) & - & - & 1 (Ref) & - & - \\
\hline & Present & 1.84 & $1.23-2.76$ & 0.003 & 2.03 & $1.35-3.05$ & 0.001 \\
\hline \multirow{2}{*}{$\begin{array}{l}\text { Radiological } \\
\text { tumor response }\end{array}$} & Present (PR) & 1 (Ref) & - & - & - & - & - \\
\hline & Absent (SD/PD) & 1.63 & $1.08-2.46$ & 0.019 & & & \\
\hline$\triangle \mathrm{MoRAL}$ & $\begin{array}{l}\text { MoRAL-non-increase } \\
\text { MoRAL-increase }\end{array}$ & - & - & - & $\begin{array}{c}1 \text { (Ref) } \\
2.18\end{array}$ & $\begin{array}{c}- \\
1.37-3.46\end{array}$ & $\begin{array}{c}- \\
0.001\end{array}$ \\
\hline
\end{tabular}

Abbreviations: SD—stable disease; PD—progressive disease; PR—partial response; MoRAL—model to predict tumor recurrence after living donor liver transplantation. ${ }^{a}$ By Cox proportional hazard model ${ }^{\mathrm{b}}$ Evaluated before the second session of TACE according to EASL criteria.

\subsection{External Validation in the Hospital and Nationwide Validation Cohorts}

In the hospital validation cohort, 93 patients $(66.4 \%)$ died during the study period (median follow-up duration $=86.6$ months $(\mathrm{IQR}=72.6-105.9)$ ) and the median OS was 21.1 months (IQR $=8.9-41.0$ months). In the nationwide validation cohort, 105 patients $(70.5 \%)$ died during the study period (median follow-up duration $=63.7$ months, IQR $=40.5-82.2$ months) and the median OS was 26.0 months (IQR $=14.3-42.3$ months). The baseline MoRAL score was not associated with OS in both the hospital and nationwide validation cohorts (Supplementary Figure S3). In the hospital 
validation cohort, the OS of the patients with MoRAL-increase was significantly shorter than that of patients with MoRAL-non-increase ( $\mathrm{HR}=1.99,95 \% \mathrm{CI}=1.28-3.09, p=0.002)$. The median OS times of MoRAL-increase and MoRAL-non-increase patients were 16.7 months ( $95 \% \mathrm{CI}=7.2-23.1$ months) and 35.4 months ( $95 \% \mathrm{CI}=31.1-40.8$ months), respectively (Figure 2A). We also divided patients in the hospital validation cohort according to baseline MoRAL score with a cutoff value of 89.5. The $\triangle$ MoRAL was not associated with $\mathrm{OS}(\mathrm{HR}=1.53,95 \% \mathrm{CI}=0.64-3.67, p=0.313)$ in patients with a low baseline MoRAL score ( $<89.5$, Figure $2 B$ ). However, $\triangle$ MoRAL showed a strong predictive performance for OS in patients with a high baseline MoRAL score $(\geq 89.5)$ (MoRAL-increase vs. MoRAL-non-increase: $\mathrm{HR}=2.90,95 \% \mathrm{CI}=1.41-5.95, p<0.001)$. The median OS was 8.1 months $(95 \% \mathrm{CI}=2.6-18.4$ months) in patients with MoRAL-increase and 35.7 months $(95 \% \mathrm{CI}=31.0-43.8$ months $)$ in those with MoRAL-non-increase (Figure 2C).
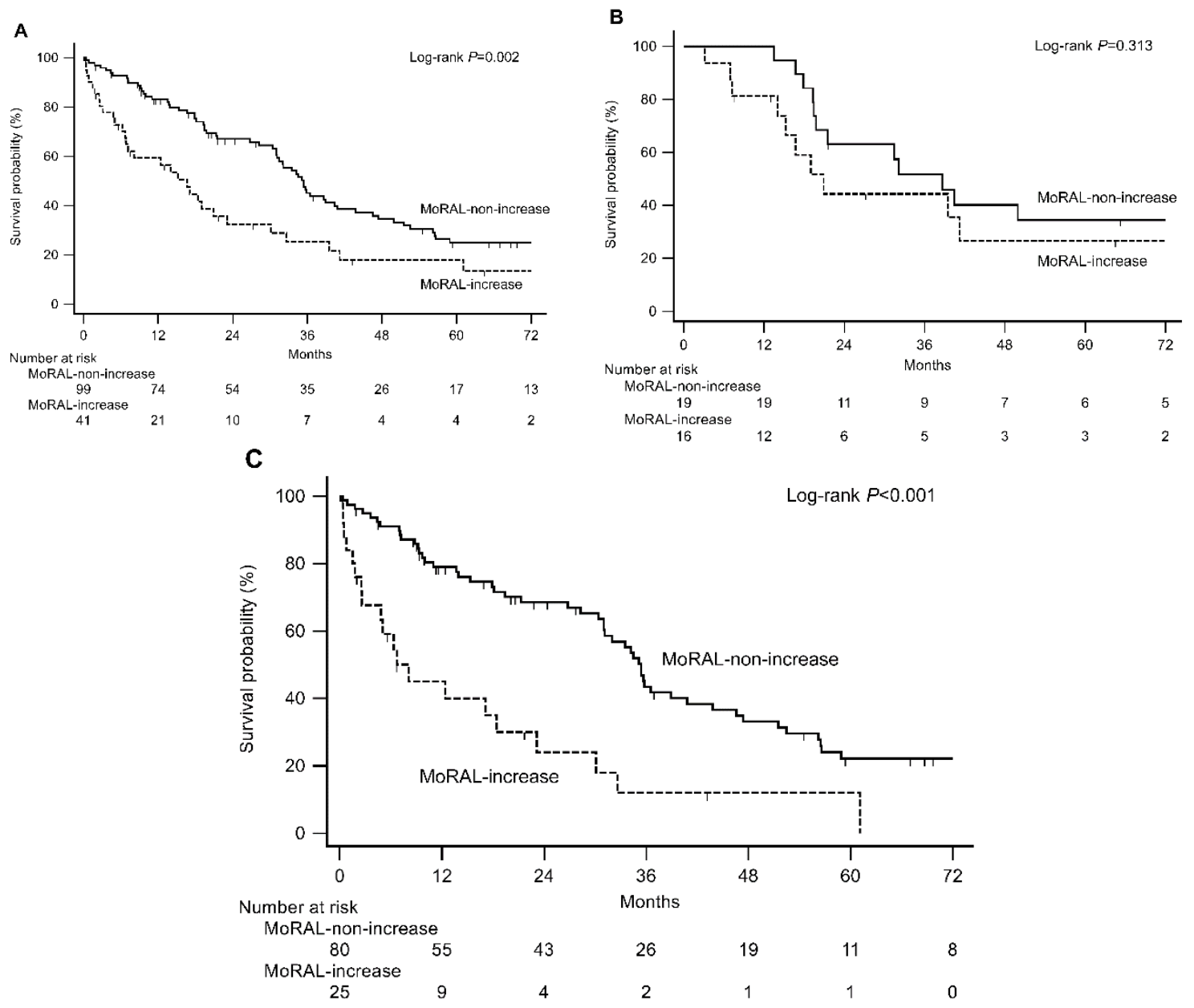

Figure 2. Prognostic significance of $\triangle \mathrm{MoRAL}$ among the entire study population (A) and patients with baseline MoRAL score $\leq 89.5$ (B) and $>89.5($ C) in the hospital validation cohort.

\subsection{Comparison with Other Indices}

As shown in Table 4, the performance of $\triangle \mathrm{MoRAL}$ in predicting OS was significantly better than the performances of initial respective serum tumor markers, baseline MoRAL score, and changes in serum tumor markers, except for the baseline AFP level, in both the training and hospital validation cohorts (all $p<0.05)$. The $\triangle$ MoRAL showed a higher $c$-index than the baseline serum AFP level in the hospital validation cohort, without statistical significance $(p=0.17)$. The $\triangle$ MoRAL also showed a higher $c$-index than the radiological tumor response and ART score of 2.5 in both cohorts, albeit without statistical significance (all $p>0.05$ ). 
Table 4. Comparison between $\triangle \mathrm{MoRAL}$ and other models for predicting overall survival.

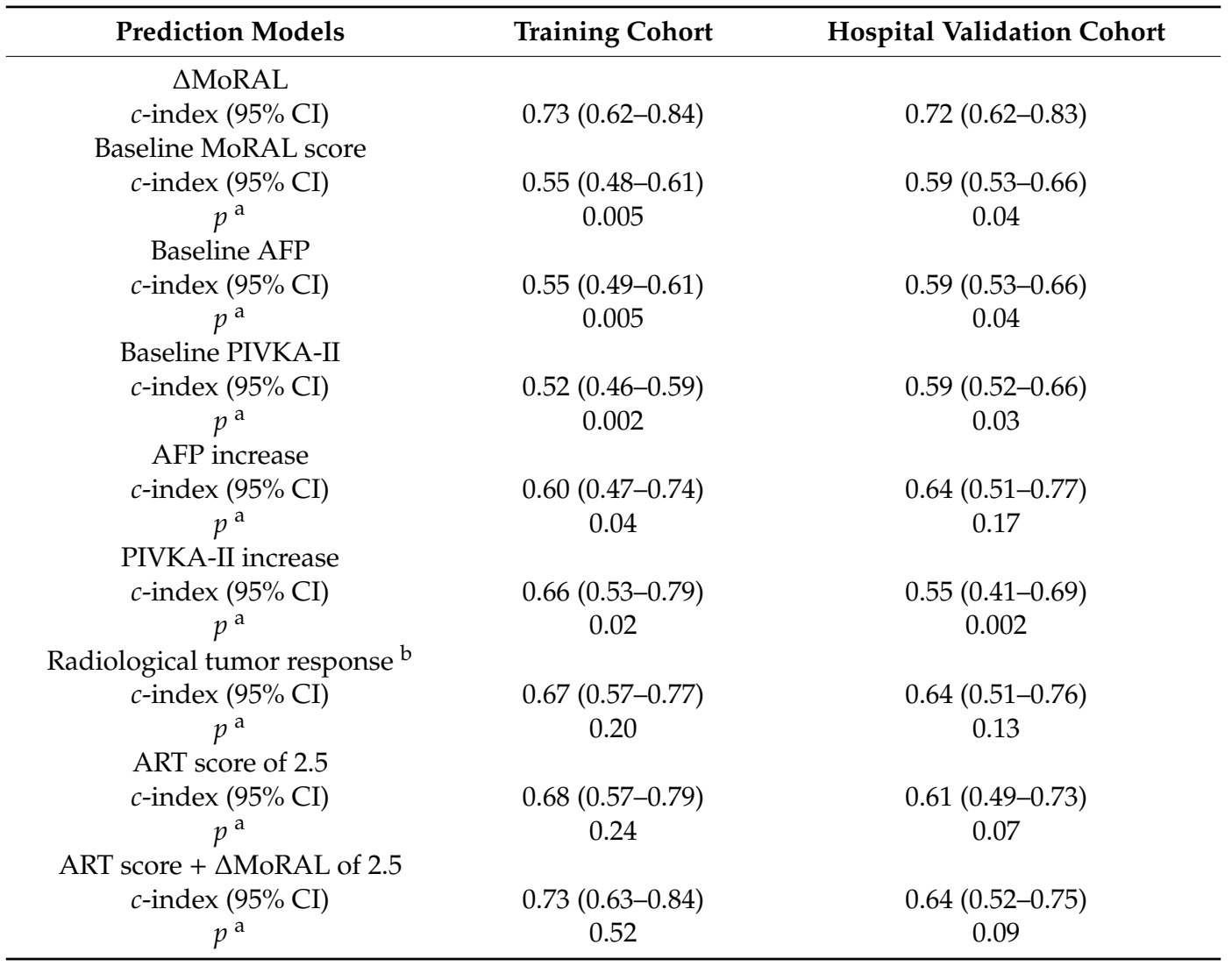

Abbreviations: MoRAL-model to predict tumor recurrence after living donor liver transplantation; AFP-alpha-fetoprotein; PIVKA-II-protein induced by vitamin $\mathrm{K}$ absence-II. ${ }^{a}$ Compared to the $c$-index of $\triangle$ MoRAL $^{\text {b }}$ Evaluated before the second session of TACE according to EASL criteria.

\section{Discussion}

In this study, we tried to find an algorithm to guide decision-making for retreatment with TACE in patients with intermediate-stage HCC using the MoRAL score. We derived and validated the MoRAL score-based algorithm using data from three large-volume hospitals and a nationwide cancer sampling registry in Korea. Although the baseline MoRAL score did not show a prognostic impact on OS, $\triangle$ MoRAL was able to differentiate two prognostic subgroups. Moreover, the prognostic impact of $\triangle$ MoRAL was more pronounced in patients with a high baseline MoRAL score $(\geq 89.5)$. Patients with a high baseline MoRAL score ( $\geq 89.5)$ and MoRAL-increase between the first and second TACE sessions had a dismal prognosis (median OS $<10$ months) in both the training and validation cohorts.

The ART score [7] and ABCR score [8] are the most popular models currently to guide decision-making for retreatment with TACE. The ART score is calculated on the basis of three parameters (increase in Child-Pugh score, increase in aspartate aminotransferase by $>25 \%$ from baseline, and tumor response). The ART score is calculated before performing a second TACE, and, on the basis of this score, patients can be differentiated into two groups, each with a distinct prognosis. For patients with an ART score of 2.5 or higher, further TACE sessions may not provide additional survival benefit and switching to sorafenib should be considered. The ABCR score was proposed by a French group. The ABCR score includes four parameters (BCLC stage and AFP at baseline, increase in Child-Pugh score, and absence of radiologic response). Patients with an ABCR score of four or higher prior to the second TACE may not benefit from further TACE sessions. However, these scoring systems have not been validated in retrospective validation studies [9-12] and may be vulnerable to bias regarding the subjective measurement of the radiologic tumor response $[19,20]$. 
The MoRAL score is calculated from serum AFP and PIVKA-II, and shows refined prognostication for tumor recurrence after LT. HCC patients with high serum AFP $(>400 \mathrm{ng} / \mathrm{mL})$ had a poor outcome and showed significant upregulation of signaling pathways-not only VEGF but also IGF1R, NOTCH, and mTOR [22]. Serum PIVKA-II has been reported to promote HCC invasion by promoting epithelial-mesenchymal transition [23]. The MoRAL score was deemed to reflect tumor biology and stemness precisely because it accurately predicts tumor recurrence after eradication of all intrahepatic visible tumors when using immunosuppressive agents, which could facilitate tumor regrowth [24]. Moreover, a higher MoRAL score was well correlated with histological findings associated with worse prognosis, such as high Edmonson-Steiner nuclear grade, microvascular invasion, bile duct invasion, perineural invasion, and serosal invasion [21]. In addition, a high MoRAL score was associated with cytokeratin 19 expression, which is a stemness-related marker and reflects tumor invasiveness [25-27], among patients who underwent surgical resection for HCC [28]. Therefore, we attempted to verify the usefulness of the original MoRAL score, assuming that the baseline MoRAL score would be helpful as well in the decision-making process for retreatment with TACE. However, unlike the HCC patients receiving LT, which is a curative treatment option, the baseline MoRAL score was not associated with OS in HCC patients receiving TACE in the present study. Moreover, variables of baseline tumor characteristics such as size and number of tumors were not associated with OS, which was consistent with the findings of two previous studies from which the ART and ABCR scores were derived $[7,8]$. These results indicated that the prognosis of patients with intermediate-stage HCC undergoing TACE relies more significantly on the treatment response, rather than the baseline tumor characteristics. In our study, the treatment response after TACE was investigated through both $\triangle$ MoRAL and the radiological tumor response. The $\triangle \mathrm{MoRAL}$ score had a significant correlation with radiological tumor response, showing a higher $c$-index than the radiological tumor response.

The prognostic impact of $\triangle$ MoRAL was more pronounced in patients with a high baseline MoRAL score $(\geq 89.5,25$ th percentile and higher). We used a different cutoff value in the current study than was used in the previous study (314.8 in the original MoRAL study by Lee et al.) [21], since the potency of treatment modalities (TACE vs. LT) is quite different. Although the baseline MoRAL score itself was not a predictor of OS, it was useful for detecting patients with poor expected OS in conjunction with $\triangle$ MoRAL. For patients with a high baseline MoRAL score $(\geq 89.5)$ followed by MoRAL-increase between the first and second TACE sessions, the expected OS was shorter than 10 months despite further TACE sessions. The OS of the patients in this group was as poor as the OS of the BCLC B control (placebo) group in the Sorafenib Hepatocellular Carcinoma Assessment Randomized Protocol trial (11.4 months) [4]. This short-expected OS implied that further TACE sessions may not provide an additional survival benefit in this group. Therefore, switching to other evidence-based systemic treatments such as sorafenib therapy should be considered in this group of patients [4-6]. Moreover, immune checkpoint inhibitors with a promising treatment response in advanced-stage HCC, such as nivolumab [29] and pembrolizumab [30], might be applied in this group of patients (i.e., baseline MoRAL score of $\geq 89.5$ with MoRAL-increase).

There are several limitations to the present study. First, because of the retrospective nature of this study, it was prone to selection bias. Approximately one-fifth of the patients satisfying the inclusion criteria were excluded because of incomplete laboratory results for tumor markers in the training cohort. However, we have shown that the performance of the MoRAL score-based algorithm was reproducible in the independent external validation cohort. Second, because Korea is a hepatitis B-endemic area [31], approximately $70 \%$ of the cases of HCC in our study population were caused by hepatitis B virus infection. Our results need to be further validated in areas with different underlying etiologies of HCC worldwide, especially in Western countries where hepatitis $C$ virus is not a major cause of HCC [32]. Third, in patients with a low baseline MoRAL score $(\leq 89.5), \triangle$ MoRAL did not predict OS. However, the median OS of patients with a low baseline MoRAL score $(\leq 89.5)$ in the training cohort, hospital validation cohort, and nationwide validation cohort were 42.7, 32.1, and 38.0 months, respectively, which indicated good prognoses. Therefore, further TACE sessions could be recommended regardless 
of $\triangle \mathrm{MoRAL}$ in these subgroups. Fourth, our prediction model did not reflect liver function, such as the baseline Child-Pugh class or Child-Pugh increase. Due to the different characteristics and dynamics between the two cohorts, prediction models that reflect both liver function and $\triangle \mathrm{MoRAL}$ in the training cohort were not validated in the hospital validation cohort. However, $\triangle$ MoRAL consistently showed good predictive performance of OS in both cohorts. Moreover, $\triangle \mathrm{MoRAL}$ tended to have a better predictive performance for OS than the ART score, which is a calculated index including liver function.

\section{Material and Methods}

\subsection{Patients}

Patients who had undergone TACE for treatment-naïve intermediate-stage HCC at Seoul National University Hospital (SNUH; Seoul, Korea) from January 2012 to June 2017 were eligible for this retrospective cohort study as a training cohort. The diagnosis of HCC was made by histology or dynamic imaging (computed tomography (CT) or magnetic resonance imaging (MRI) scans) $[13,14]$. Patients aged between 20 and 80 years at the time of the first TACE, who underwent at least two TACE sessions within 90 days, were included for analysis. All enrolled patients had no evidence of extrahepatic metastasis. Patients who had incomplete data of tumor markers or who received TACE as a bridge treatment for LT or for down-staging treatment before resection were excluded.

Two external validation cohorts were established: the hospital validation cohort and the nationwide validation cohort. The hospital validation cohort consisted of patients treated at the Samsung Medical Center (SMC; Seoul, Korea) and Ewha Womans University Medical Center (EUMC; Seoul, Korea) between February 2009 and December 2012, with the same inclusion/exclusion criteria. The nationwide validation cohort was acquired from the Korean Primary Liver Cancer Registry between 2008 and 2014, with the same inclusion/exclusion criteria (Supplementary Methods).

This study was conducted in accordance with the World Medical Association Declaration of Helsinki and was approved by the Institutional Review Board of each participating center (SNUH No. 1702-067-831, SMC No. 2018-08-137, and EUMC No. 2016-07-081-015), and by the Korean Central Cancer Registry, Ministry of Health and Welfare Korea, and the Korean Liver Cancer Association.

\subsection{Treatment, Procedures and Assessments}

Baseline laboratory and radiological findings of the enrolled patients in the training cohort and validation cohorts were assessed within seven days before the first TACE session. The MoRAL score was calculated as follows: $11 \times \sqrt{ }$ PIVKA $+2 \times \sqrt{ }$ AFP [21]. To measure the changes in MoRAL score $(\triangle M o R A L)$, the MoRAL score was calculated at two time points. The baseline MoRAL score was calculated within seven days before the first TACE session. The second MoRAL score was recalculated one day before the second TACE session. The $\triangle$ MoRAL scores were found by comparing the second score with the baseline MoRAL score for each patient. We defined a MoRAL-increase when the second MoRAL score increased by $\geq 5 \%$ compared to the baseline MoRAL score, whereas a MoRAL-non-increase was defined by the second MoRAL score increasing by $<5 \%$ or decreasing compared to the baseline MoRAL score.

Super selective TACE was performed by experienced interventional radiologists $(>10$ years of experience) at the participating centers according to tumor distribution and underlying liver function, as described elsewhere (Supplementary Methods) [33-36]. After a maximum of 90 days from the initial TACE session, dynamic imaging was performed to evaluate tumor response and repeated TACE was done when residual or newly developed tumors were observed without the presence of Child-Pugh C liver function or Eastern Cooperative Oncology Group performance status $\geq 2$.

Radiological tumor response was assessed by dynamic CT or MRI scans prior to the second TACE session, according to the European Association for the Study of the Liver (EASL) criteria (Supplementary Methods) [37]. We defined partial response (PR) as the presence of a tumor response, and stable disease (SD) and progressive disease (PD) as the absence of a tumor response. Patients with 
complete response (CR) were not included in this study because they did not receive further TACE sessions after the first TACE within 90 days.

\subsection{Endpoint}

The primary endpoint was OS, which was measured from the day prior to the second TACE session until death from any cause or last follow-up. The survival data of the enrolled patients were obtained from the national statistical data from the Korean Ministry of Government Administration and Home Affairs. The data cutoff dates were 31 August 2018 in the training cohort and hospital validation cohort, and 31 December 2016 in the nationwide validation cohort.

\subsection{Statistical Analysis}

Kaplan-Meier curves were generated for OS and the log-rank test was used for group comparisons. A multivariable stepwise Cox regression analysis was performed to find independent predictors of OS. The Harrell concordance $(c)$-index was calculated to compare the discriminatory abilities of $\triangle \mathrm{MoRAL}$ and other indices for predicting OS. The statistical analyses were performed using IBM SPSS Statistics for Windows, version 23.0 (IBM Corp., Armonk, NY) and R language, version 3.5.2 (R Foundation for Statistical Computing, Vienna, Austria). All statistical tests were 2-sided and $P$ values less than 0.05 were considered statistically significant.

\section{Conclusions}

In conclusion, we have derived and validated the MoRAL score-based algorithm for predicting OS after the first TACE session for patients with intermediate-stage HCC, which is based on objective and reproducible predictors. This algorithm might provide refined prognostication for predicting OS after TACE. Specifically, patients with a high baseline MoRAL score $(\geq 89.5)$ and MoRAL-increase between the first and second sessions of TACE had a dismal prognosis, and might be potential candidates for systemic therapy. Future clinical trials directly comparing repeated TACE and systemic therapy are warranted to verify the clinical efficacy of this algorithm.

Supplementary Materials: The following are available online at http://www.mdpi.com/2072-6694/11/11/1721/s1, Figure S1: CONSORT diagrams of the training cohort; Figure S2: Correlation between each radiologic tumor response and the $\triangle$ MoRAL percentage; Figure S3: Prognostic significance of the baseline MoRAL score of 314.8 in the hospital validation cohort. Table S1: Multivariable analysis of prognostic factors in the training cohort excluding patients with alcoholic liver disease $(n=173)$.

Author Contributions: Study concept and design: J.-H.L. and H.Y.K., Provision of study patients and acquisition of clinical data: J.S.Y., D.H.S., H.Y.K., J.-H.L., C.-H.L., S.W.K., H.Y.L., J.Y.N., Y.C., Y.B.L., E.J.C., S.J.Y., H.-C.K., J.W.C., Y.J.K., and J.-H.L., Biostatistical analysis and interpretation: J.S.Y. and J.-H.L., Drafting of the manuscript: J.S.Y. and J.-H.L., Critical revision of the manuscript for important intellectual content: D.H.S., H.Y.K., J.-H.L., Y.J.K., and J.-H.L., Final approval of manuscript: all authors.

Funding: This work was supported by grants from the National Research Foundation of Korea (NRF) funded by the Korea government (MSIP) (No. 2019R1A2C2010311), from the Seoul National University Hospital Research Fund (03-2016-0380), and from Liver Research Foundation of Korea as part of Bio Future Strategies Research Project. Funding sources had no involvement in study design, in the collection, analysis and interpretation of data, in the writing of the report, and in the decision to submit the article for publication. The corresponding authors had full access to all study data and had final responsibility for the decision to submit for publication.

Acknowledgments: The database used in this study was provided by the Korean Central Cancer Registry, Ministry of Health and Welfare, South Korea, and the Korean Liver Cancer Association.

Conflicts of Interest: Jeong-Hoon Lee reports receiving lecture fee from GreenCross Cell, Daewoong Pharmaceuticals, and Gilead Korea; Su Jong Yu reports lecture fee from Bayer HealthCare Pharmaceuticals; Yoon Jun Kim, research grants from Bristol-Myers Squibb, Roche, J.W. Creagene, Bukwang Pharmaceuticals, Handok Pharmaceuticals, Hanmi Pharmaceuticals, Yuhan Pharmaceuticals, Samjin Pharmaceuticals, and Pharmaking, and lecture fees from Bayer HealthCare Pharmaceuticals, Gilead Science, MSD Korea, Yuhan Pharmaceuticals, Samil Pharmaceuticals, CJ Pharmaceuticals, Bukwang Pharmaceuticals, and Handok Pharmaceuticals; Jung-Hwan Yoon, research grants from AstraZeneca, Bayer HealthCare Pharmaceuticals, Daewoong Pharmaceuticals, and Bukwang Pharmaceuticals. No other potential conflict of interest relevant to this article was reported. 


\section{References}

1. Akinyemiju, T.; Abera, S.; Ahmed, M.; Alam, N.; Alemayohu, M.A.; Allen, C.; Al-Raddadi, R.; Alvis-Guzman, N.; Amoako, Y.; Artaman, A.; et al. The Burden of Primary Liver Cancer and Underlying Etiologies From 1990 to 2015 at the Global, Regional, and National Level: Results From the Global Burden of Disease Study 2015. JAMA Oncol. 2017, 3, 1683-1691. [CrossRef] [PubMed]

2. Forner, A.; Reig, M.E.; de Lope, C.R.; Bruix, J. Current strategy for staging and treatment: the BCLC update and future prospects. Semin. Liver Dis. 2010, 30, 61-74. [CrossRef] [PubMed]

3. Llovet, J.M.; Bru, C.; Bruix, J. Prognosis of hepatocellular carcinoma: the BCLC staging classification. Semin. Liver Dis. 1999, 19, 329-338. [CrossRef] [PubMed]

4. Bruix, J.; Raoul, J.L.; Sherman, M.; Mazzaferro, V.; Bolondi, L.; Craxi, A.; Galle, P.R.; Santoro, A.; Beaugrand, M.; Sangiovanni, A.; et al. Efficacy and safety of sorafenib in patients with advanced hepatocellular carcinoma: subanalyses of a phase III trial. J. Hepatol. 2012, 57, 821-829. [CrossRef] [PubMed]

5. Llovet, J.M.; Ricci, S.; Mazzaferro, V.; Hilgard, P.; Gane, E.; Blanc, J.F.; de Oliveira, A.C.; Santoro, A.; Raoul, J.L.; Forner, A.; et al. Sorafenib in advanced hepatocellular carcinoma. New Engl. J. Med. 2008, 359, 378-390. [CrossRef] [PubMed]

6. Galle, P.R.; Tovoli, F.; Foerster, F.; Worns, M.A.; Cucchetti, A.; Bolondi, L. The treatment of intermediate stage tumours beyond TACE: From surgery to systemic therapy. J. Hepatol. 2017, 67, 173-183. [CrossRef] [PubMed]

7. Sieghart, W.; Hucke, F.; Pinter, M.; Graziadei, I.; Vogel, W.; Muller, C.; Heinzl, H.; Trauner, M.; Peck-Radosavljevic, M. The ART of decision making: retreatment with transarterial chemoembolization in patients with hepatocellular carcinoma. Hepatology (Baltim. Md.) 2013, 57, 2261-2273. [CrossRef]

8. Adhoute, X.; Penaranda, G.; Naude, S.; Raoul, J.L.; Perrier, H.; Bayle, O.; Monnet, O.; Beaurain, P.; Bazin, C.; Pol, B.; et al. Retreatment with TACE: the ABCR SCORE, an aid to the decision-making process. J. Hepatol. 2015, 62, 855-862. [CrossRef]

9. Kudo, M.; Arizumi, T.; Ueshima, K. Assessment for retreatment (ART) score for repeated transarterial chemoembolization in patients with hepatocellular carcinoma. Hepatology (Baltim. Md.) 2014, 59, $2424-2425$. [CrossRef]

10. Arizumi, T.; Ueshima, K.; Iwanishi, M.; Minami, T.; Chishina, H.; Kono, M.; Takita, M.; Kitai, S.; Inoue, T.; Yada, N.; et al. Evaluation of ART Scores for Repeated Transarterial Chemoembolization in Japanese Patients with Hepatocellular Carcinoma. Oncology 2015, 89 (Suppl. 2), 4-10. [CrossRef]

11. Facciorusso, A.; Bhoori, S.; Sposito, C.; Mazzaferro, V. Repeated transarterial chemoembolization: An overfitting effort? J. Hepatol. 2015, 62, 1440-1442. [CrossRef] [PubMed]

12. Kloeckner, R.; Pitton, M.B.; Dueber, C.; Schmidtmann, I.; Galle, P.R.; Koch, S.; Worns, M.A.; Weinmann, A. Validation of Clinical Scoring Systems ART and ABCR after Transarterial Chemoembolization of Hepatocellular Carcinoma. J. Vasc. Int. Rad. 2017, 28, 94-102. [CrossRef] [PubMed]

13. Marrero, J.A.; Kulik, L.M.; Sirlin, C.B.; Zhu, A.X.; Finn, R.S.; Abecassis, M.M.; Roberts, L.R.; Heimbach, J.K. Diagnosis, Staging, and Management of Hepatocellular Carcinoma: 2018 Practice Guidance by the American Association for the Study of Liver Diseases. Hepatology (Baltim. Md.) 2018, 68, 723-750. [CrossRef] [PubMed]

14. European Association for the Study of the Liver. EASL Clinical Practice Guidelines: Management of hepatocellular carcinoma. J. Hepatol. 2018, 69, 182-236. [CrossRef] [PubMed]

15. Choi, J.; Shim, J.H.; Shin, Y.M.; Kim, K.M.; Lim, Y.S.; Lee, H.C. Clinical significance of the best response during repeated transarterial chemoembolization in the treatment of hepatocellular carcinoma. J. Hepatol. 2014, 60, 1212-1218. [CrossRef]

16. Jung, E.S.; Kim, J.H.; Yoon, E.L.; Lee, H.J.; Lee, S.J.; Suh, S.J.; Lee, B.J.; Seo, Y.S.; Yim, H.J.; Seo, T.S.; et al. Comparison of the methods for tumor response assessment in patients with hepatocellular carcinoma undergoing transarterial chemoembolization. J. Hepatol. 2013, 58, 1181-1187. [CrossRef]

17. Prajapati, H.J.; Spivey, J.R.; Hanish, S.I.; El-Rayes, B.F.; Kauh, J.S.; Chen, Z.; Kim, H.S. mRECIST and EASL responses at early time point by contrast-enhanced dynamic MRI predict survival in patients with unresectable hepatocellular carcinoma (HCC) treated by doxorubicin drug-eluting beads transarterial chemoembolization (DEB TACE). Ann. Oncol. 2013, 24, 965-973. [CrossRef]

18. Gillmore, R.; Stuart, S.; Kirkwood, A.; Hameeduddin, A.; Woodward, N.; Burroughs, A.K.; Meyer, T. EASL and mRECIST responses are independent prognostic factors for survival in hepatocellular cancer patients treated with transarterial embolization. J. Hepatol. 2011, 55, 1309-1316. [CrossRef] 
19. Ronot, M.; Bouattour, M.; Wassermann, J.; Bruno, O.; Dreyer, C.; Larroque, B.; Castera, L.; Vilgrain, V.; Belghiti, J.; Raymond, E.; et al. Alternative Response Criteria (Choi, European association for the study of the liver, and modified Response Evaluation Criteria in Solid Tumors [RECIST]) Versus RECIST 1.1 in patients with advanced hepatocellular carcinoma treated with sorafenib. Oncology 2014, 19, 394-402. [CrossRef]

20. Donati, O.F.; Do, R.K.; Hotker, A.M.; Katz, S.S.; Zheng, J.; Moskowitz, C.S.; Beattie, C.; Brown, K.T. Interreader and inter-test agreement in assessing treatment response following transarterial embolization for hepatocellular carcinoma. Eur. Radiol. 2015, 25, 2779-2788. [CrossRef]

21. Lee, J.H.; Cho, Y.; Kim, H.Y.; Cho, E.J.; Lee, D.H.; Yu, S.J.; Lee, J.W.; Yi, N.J.; Lee, K.W.; Kim, S.H.; et al. Serum Tumor Markers Provide Refined Prognostication in Selecting Liver Transplantation Candidate for Hepatocellular Carcinoma Patients Beyond the Milan Criteria. Ann. Surg. 2016, 263, 842-850. [CrossRef] [PubMed]

22. Montal, R.; Andreu-Oller, C.; Bassaganyas, L.; Esteban-Fabro, R.; Moran, S.; Montironi, C.; Moeini, A.; Pinyol, R.; Peix, J.; Cabellos, L.; et al. Molecular portrait of high alpha-fetoprotein in hepatocellular carcinoma: implications for biomarker-driven clinical trials. Br. J. Cancer 2019. [CrossRef] [PubMed]

23. Xu, J.F.; Liu, X.Y. PIVKA-II is an independent prognostic factor for overall survival of HCC patients and maybe associated with epithelial-mesenchymal transition. J. Hepatol. 2015, 63, 1040-1041. [CrossRef] [PubMed]

24. Rodriguez-Peralvarez, M.; Tsochatzis, E.; Naveas, M.C.; Pieri, G.; Garcia-Caparros, C.; O’Beirne, J.; Poyato-Gonzalez, A.; Ferrin-Sanchez, G.; Montero-Alvarez, J.L.; Patch, D.; et al. Reduced exposure to calcineurin inhibitors early after liver transplantation prevents recurrence of hepatocellular carcinoma. J. Hepatol. 2013, 59, 1193-1199. [CrossRef]

25. Hoshida, Y.; Toffanin, S.; Lachenmayer, A.; Villanueva, A.; Minguez, B.; Llovet, J.M. Molecular classification and novel targets in hepatocellular carcinoma: recent advancements. Semin. Liver Dis. 2010, 30, 35-51. [CrossRef] [PubMed]

26. Yoneda, N.; Sato, Y.; Kitao, A.; Ikeda, H.; Sawada-Kitamura, S.; Miyakoshi, M.; Harada, K.; Sasaki, M.; Matsui, O.; Nakanuma, Y. Epidermal growth factor induces cytokeratin 19 expression accompanied by increased growth abilities in human hepatocellular carcinoma. Lab. Investig. 2011, 91, 262-272. [CrossRef] [PubMed]

27. Yamamoto, T.; Uenishi, T.; Ogawa, M.; Ichikawa, T.; Hai, S.; Sakabe, K.; Tanaka, S.; Kato, H.; Mikami, S.; Ikebe, T.; et al. Immunohistologic attempt to find carcinogenesis from hepatic progenitor cell in hepatocellular carcinoma. Dig. Surg. 2005, 22, 364-370. [CrossRef]

28. Lee, H.Y.; Lee, Y.B.; Lee, J.H.; Chung, S.W.; Kim, M.S.; Kim, S.W.; Yoon, J.S.; Chang, Y.; Cho, E.J.; Yu, S.J.; et al. O-038: A MoRAL Score Utilizing Serum Tumor Markers Provides Refined Prognostication of Patients with Hepatocellular Carcinoma after Curative Resection: Data from 662 Consecutive Patients. 춘 추 계 학 술 대회 (KASL) 2018, 2018, 29-30.

29. El-Khoueiry, A.B.; Sangro, B.; Yau, T.; Crocenzi, T.S.; Kudo, M.; Hsu, C.; Kim, T.Y.; Choo, S.P.; Trojan, J.; Welling, T.H.R.; et al. Nivolumab in patients with advanced hepatocellular carcinoma (CheckMate 040): an open-label, non-comparative, phase $1 / 2$ dose escalation and expansion trial. Lancet (Lond. Engl.) 2017, 389, 2492-2502. [CrossRef]

30. Zhu, A.X.; Finn, R.S.; Edeline, J.; Cattan, S.; Ogasawara, S.; Palmer, D.; Verslype, C.; Zagonel, V.; Fartoux, L.; Vogel, A.; et al. Pembrolizumab in patients with advanced hepatocellular carcinoma previously treated with sorafenib (KEYNOTE-224): a non-randomised, open-label phase 2 trial. Lancet. Oncol. 2018, 19, 940-952. [CrossRef]

31. Cho, E.J.; Kim, S.E.; Suk, K.T.; An, J.; Jeong, S.W.; Chung, W.J.; Kim, Y.J. Current status and strategies for hepatitis B control in Korea. Clin. Mol. Hepatol. 2017, 23, 205-211. [CrossRef] [PubMed]

32. El-Serag, H.B.; Rudolph, K.L. Hepatocellular carcinoma: epidemiology and molecular carcinogenesis. Gastroenterology 2007, 132, 2557-2576. [CrossRef] [PubMed]

33. Shin, S.W.; Do, Y.S.; Choo, S.W.; Lieu, W.C.; Cho, S.K.; Park, K.B.; Yoo, B.C.; Kang, E.H.; Choo, I.W. Diaphragmatic weakness after transcatheter arterial chemoembolization of inferior phrenic artery for treatment of hepatocellular carcinoma. Radiology 2006, 241, 581-588. [CrossRef] [PubMed]

34. Kim, H.C.; Chung, J.W.; Jae, H.J.; Yoon, J.H.; Lee, J.H.; Kim, Y.J.; Lee, H.S.; Yoon, C.J.; Park, J.H. Caudate lobe hepatocellular carcinoma treated with selective chemoembolization. Radiology 2010, 257, 278-287. [CrossRef] [PubMed] 
35. Takayasu, K.; Arii, S.; Kudo, M.; Ichida, T.; Matsui, O.; Izumi, N.; Matsuyama, Y.; Sakamoto, M.; Nakashima, O.; $\mathrm{Ku}, \mathrm{Y}$; et al. Superselective transarterial chemoembolization for hepatocellular carcinoma. Validation of treatment algorithm proposed by Japanese guidelines. J. Hepatol. 2012, 56, 886-892. [CrossRef]

36. Lee, S.; Kim, K.A.; Park, M.S.; Choi, S.Y. MRI Findings and Prediction of Time to Progression of Patients with Hepatocellular Carcinoma Treated with Drug-eluting Bead Transcatheter Arterial Chemoembolization. J. Korean Med Sci. 2015, 30, 965-973. [CrossRef]

37. Bruix, J.; Sherman, M.; Llovet, J.M.; Beaugrand, M.; Lencioni, R.; Burroughs, A.K.; Christensen, E.; Pagliaro, L.; Colombo, M.; Rodes, J. Clinical management of hepatocellular carcinoma. Conclusions of the Barcelona-2000 EASL conference. European Association for the Study of the Liver. J. Hepatol. 2001, 35, 421-430. [CrossRef]

(C) 2019 by the authors. Licensee MDPI, Basel, Switzerland. This article is an open access article distributed under the terms and conditions of the Creative Commons Attribution (CC BY) license (http://creativecommons.org/licenses/by/4.0/). 